\title{
Performance evaluation of non-persistent CSMA as anti-collision protocol for active RFID tags
}

E. Egea-López, J. Vales-Alonso, A. S. Martínez-Sala, M. V. Bueno-Delgado, and J. García-Haro

Department of Information Technologies and Communications

Polytechnic University of Cartagena, Spain

\{esteban.egea, javier.vales, alejandros.martinez, mvictoria.bueno,

joang.haro\}@upct.es

Summary. In this paper we propose the use of non-persistent CSMA as an anticollision procedure for RFID active tags. Current proposals for both passive and active tags are based on the framed slotted ALOHA protocol, which does not scale well requiring additional procedures for frame length adaptation. However, active RFID devices already include carrier sense capabilities with no additional cost and, thus, CSMA may be employed seamlessly. Nevertheless, selecting the contention micro-slots of CSMA in the classical way (i.e., with a uniform distribution and an exponential back-off algorithm) does not result in an efficient identification process, as we will demonstrate. Fortunately, better choices can be found. Recently, an optimal distribution for the selection of micro-slots for event-driven sensor networks has been computed, as well as a practical implementation: the Sift distribution. In this work we propose the application of the quasi-optimal Sift distribution along with CSMA for active tag identification. By means of an analytical study, we evaluate the average time needed for identification with this mechanism and compare it with the current ISO 18000-7 and EPC "Gen 2" standard. The results reveal that the Sift-based non-persistent CSMA outperforms both of them. Moreover, it also scales much better, without the need for further adaptation mechanisms.

Key words: Radio Frequency Identification (RFID), anti-collision protocol, non-persistent CSMA, EPC "Gen 2", ISO 18000-7, performance evaluation, active RFID tag.

\section{Introduction}

Radio Frequency Identification (RFID) systems are one of the enabling technologies for the ubiquitous computing paradigm [1]. Its foreseen application range spans from replacement of bar-code systems to location of containers in 
large cargo vehicles. A wide range of RFID technologies have been in study to match such a broad range of applications. All of them share a common architecture: a basic RFID cell consists of a reader device (aka master or interrogator) and a (potentially large) set of RFID tags, which reply to the queries or enforce the commands from the interrogator. RFID devices are classified according to the source of energy of the tags: passive ones do not have a power source and obtain the energy from the reader signal (via induction), whereas active ones incorporate their own battery. On the one hand, passive tags are targeted to be inexpensive and, thus, very simple, usually read-only, devices. Their coverage typically ranges from centimetres to a couple of metres. On the other hand, active tags are more complex devices, with more sophisticated capabilities (usually integrating a microprocessor and memory) and they can be read and written from distances in excess of 100 meters [1]. Whereas passive RFID systems are the most deployed and have been studied for years $[2,3,4]$, active RFID systems have devoted little academic attention, and only recently a standard is available [5].

In both cases, the tag collision problem arises: in a RFID cell, if multiple tags are to be identified simultaneously, reply messages from tags can collide and cancel each other. Thus, an anti-collision mechanism is needed. Since, in a typical application, items (with attached tags) enter and leave the reader coverage area, the goal of this mechanism is to communicate with the tags as quickly and reliably as possible, ensuring that all tags have been identified. An additional goal for active tags is to save energy in order to maximise the battery lifetime. Therefore, the tag identification problem deals with identifying multiple objects with minimal delay and power consumption, reliability, line-of-sight independence and scalability. Unlike classical medium access protocols, channel utilisation and fairness are not usually issues in RFID systems.

For passive tags, mainly due to the limitations of the devices, the protocols are very simple and most of them fall into the following two categories [2]:

- Splitting algorithms. The set of tags to be identified is split in disjoint smaller subsets until the number of tags in a subset becomes one. It is done either by the tags selecting a random number or by the reader sending a string that matches only a subset of tags identification number (ID). Algorithms of this type can be viewed as a tree search.

- Probabilistic algorithms. The other major family of protocols is based on Framed Slotted ALOHA (FSA) [6]. In this case, after receiving a signal from the reader, the tags randomly select a slot out of $K$ (the frame length) and send their ID. This mechanism is very simple, but when the number of tags increases, it needs some mechanism to adapt the frame length $(K)$ [3].

What are the approaches used with active tags? The answer is not straightforward due to some existing confusion around this technology. On one hand, there is a lack of scientific literature that specifically addresses the collisionresolution problem for active tags. The ISO 18000-7 standard [5] deals with it 
and proposes framed slotted ALOHA as an anti-collision protocol, suggesting a frame length adaption mechanism but without specifying a particular one and leaving it open to the vendors. In addition, the EPCglobal organisation, leader of development of industry-driven standards for this field, has settled the EPC "Gen 2" as a reference standard [7]. The anti-collision procedure of "Gen 2" is supposed to be independent of the class of device, that is, passive or active. With minor changes, EPC "Gen 2" has also chosen framed slotted ALOHA and does suggest a specific algorithm for frame length adaptation.

On the other hand, a typical active tag has the capabilities of an onboard microprocessor and a sophisticated transceiver and may use Bluetooth or IEEE 802.11 protocols or Wireless Sensor Networks (WSN) MAC protocols [8]. It is clear that these protocols are designed with different requirements in mind and, at the moment, the cost of these devices is still possibly too high. Therefore, it seems that the possible choices are: very simple approaches suitable for passive tags or very sophisticated proposals designed for different purposes.

Is any of these choices efficient? In this paper we explore an intermediate solution: the use of non-persistent Carrier Sense Multiple Access (CSMA) as anti-collision mechanism for active RFID tags. With this mechanism, after receiving an identification request, nodes would listen to the channel for a randomly selected number of contention slots ${ }^{1}$. A node would transmit if the channel remains idle during this interval. Otherwise, it would defer transmission. As we will show, if micro-slots are selected uniformly (the classical approach), the identification of tags does not have to be more efficient than a framed slotted ALOHA. However, an optimised distribution for the selection of CSMA contention micro-slots has been proposed for Wireless Sensor Networks [9]. This distribution minimises the probability of collision when $N$ stations become simultaneously backlogged, which is exactly the main problem of RFID identification. Using this distribution, the identification process is faster and more scalable than the FSA proposals, and even simplifies the implementation of the protocol. It should be remarked that the use of CSMA is feasible for active RFID devices: A typical low cost chip for active RFID [1] already integrates carrier sensing capabilities without any additional cost, even though in the application domain of active RFID (vehicle and container tracking and management) it is already assumed the need for more complex and expensive tags. Moreover, it may reduce the cost compared to devices that use complex protocols like IEEE 802.11.

In this paper we support this solution by studying analytically the performance of quasi-optimal non-persistent CSMA as an anti-collision mechanism for RFID identification. We compute the average number of identification cycles needed to identify all the tags present in a coverage cell for CSMA and ISO 18000-7 or EPC "Gen 2" and compare them. The rest of this paper is

\footnotetext{
${ }^{1}$ We will refer to a contention slot as micro-slot, to distinguish it from a slotted ALOHA one.
} 
organised as follows: Section 2 briefly reviews the related work. In Section 3 the different proposals to be compared are reviewed and analysed. Section 4 provides a comparison and discussion of the proposals. Finally, section 5 concludes and outlines possible future works.

\section{Related Work}

Most of the anti-collision protocols focus on passive RFID tags [2]. In this case, the limitations of the device, usually impose the use of very simple protocols, all the burden of the identification process lying on the reader. The different proposals fall into the two following categories: (i) splitting algorithms or (ii) probabilistic protocols. In the first group, a well-known protocol, called QT memoryless [2] exemplifies its operation: the reader sends a string prefix and all the tags whose ID match that prefix reply. The reader appends a new digit to the string prefix subsequently. If there is no collision in the response, a tag has been identified. This type of algorithms can be observed as a tree search and are deterministic, meaning that all tags are identified with probability 1 within a bounded time. However, this time can be very long, depending on the length of the tag IDs.

In the second group, framed slotted ALOHA is practically a unanimous choice. For instance, the I-Code protocol [3] is used with passive tags. Surprisingly, most of the proposals for active systems have also selected this approach: the ISO 18000-7 standard [5] as well as the EPC "Gen 2" protocol [7]. The latter, which is expected to be a de facto standard, is to be used with both active and passive tags. Unlike ISO 18000-7, EPC does suggest a procedure to adapt the frame length.

Vogt [3] analyses the identification process of framed slotted ALOHA as a Markov chain and derives two procedures to dynamically adapt the frame length. It is assumed that tags are not acknowledged and all tags participate in every identification round. In this paper, we use a slightly modified analysis, considering that identified tags do not keep on participating, since the two major proposals, ISO 18000-7 and EPC "Gen 2", state that tags retire after being acknowledged.

As said in Section 1, we propose the use of CSMA with the optimal probability distribution $\left(p^{*}\right)$ for the selection of CSMA contention micro-slots derived in [9]. This distribution maximises the probability of success when $N$ stations become simultaneously backlogged, but depends on the number of slots in use $(K)$ and the number of nodes $(N)$ contending. Since the latter is usually unknown (also in RFID), an approximation is also provided, the Sift distribution, which not only keeps close to the optimal for a wide range of its configuration parameters but it is also scalable. The authors of reference [9] discuss different applications in wireless sensor networks, but RFID is not mentioned. In this paper we show that RFID is a major field of application of this optimised distribution. 
Finally, both CSMA and framed slotted ALOHA have been extensively studied [10,6], but as classical MAC protocols, focusing on the channel utilisation and access delay. In RFID, on the contrary, the appropriate performance metric is the identification delay. We evaluate the performance of the protocols regarding this metric.

\section{Analysis of proposals}

In this section, the different proposals to be compared will be reviewed and analysed.

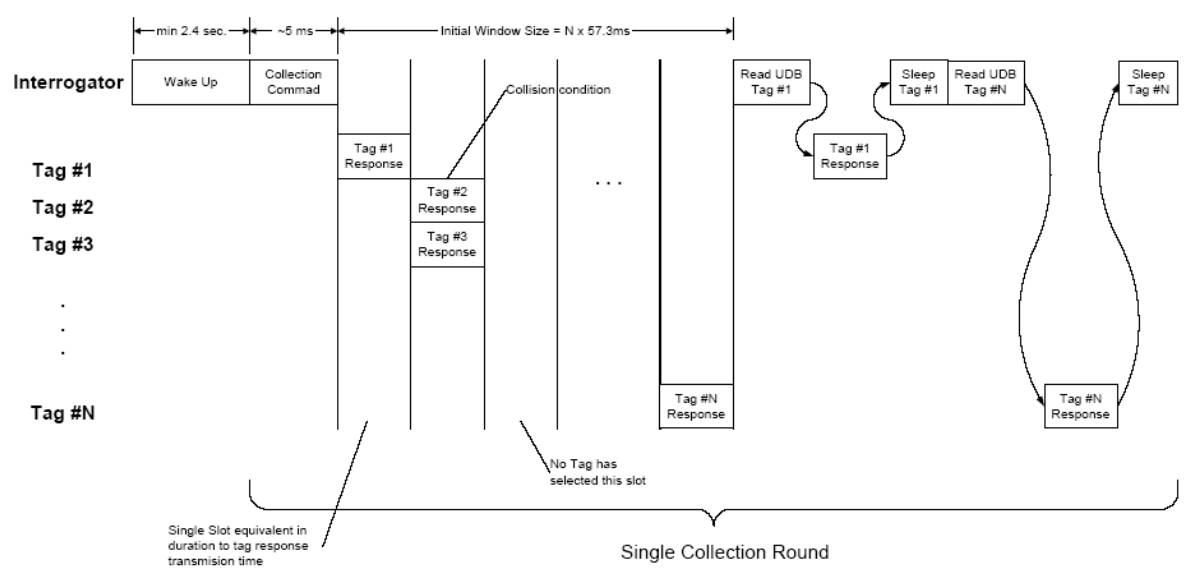

Fig. 1. Anti-collision procedure of ISO 18000-7 (from [5])

\subsection{Framed Slotted ALOHA}

Both ISO 18000-7 and EPC "Gen 2" [5, 7] define a similar anti-collision procedure that we call generically: Framed slotted ALOHA (FSA). In both cases, a population of $N$ tags start the identification process after receiving a collection command from the interrogator. At this moment, nodes randomly select a slot with a uniform distribution and transmit their ID at the selected slot. We refer to the number of possible slots to choose as frame length, $K$. If two or more nodes select the same slot, a collision occurs. For each slot with a single reply, the interrogator sends an ACK packet to put the tag to sleep, preventing it from participating again in the identification process. The acknowledged tags (already identified) withdraw from contention in the following rounds. Fig. 1 illustrates the process. We refer to a collection command plus the $K$ slots as an identification cycle. Although shown in Fig. 1, we assume no transmission 


\begin{tabular}{|c|c|c|c|c|c|c|c|c|c|c|}
\hline Slots / Tags & 10 & 20 & 30 & 40 & 50 & 60 & 70 & 80 & 90 & 100 \\
\hline 4 & 8.2 & 60 & 630 & 8159 & $1.110^{5}$ & $1.610^{6}$ & $2.510^{7}$ & $3.810^{8}$ & $6.010^{9}$ & $9.610^{10}$ \\
\hline 8 & 3.67 & 8.56 & 19.6 & 49.4 & 138.0 & 413.9 & 1304.2 & 4244.6 & 14127 & 47797 \\
\hline 16 & 2.44 & 4.11 & 6.15 & 8.93 & 13.03 & 19.3 & 29.41 & 46.0 & 73.81 & 121.3 \\
\hline 32 & 1.89 & 2.76 & 3.60 & 4.47 & 5.424 & 6.50 & 7.76 & 9.26 & 11.0 & 13.2 \\
\hline 64 & 1.54 & 2.15 & 2.61 & 3.06 & 3.465 & 3.90 & 4.32 & 4.77 & 5.23 & 5.72 \\
\hline
\end{tabular}

Table 1. Average number of identification cycles versus number of tags

of data is done and tags only identify themselves. After three collection rounds without reply, the interrogator assumes that all nodes have been identified.

As explained in [3], the identification process can be modelled as a (homogeneous) Markov process $\left\{X_{s}\right\}$, where $X_{s}$ denotes the number of tags unidentified at the $s$ identification cycle. Thus, the state space of the Markov process is $\{N, N-1, \ldots, 0\}$. The probability distribution of the random variable $\mu_{r}$ that indicates the number of slots being filled with exactly $r$ tags is:

$$
P_{K, N}\left(\mu_{r}=m\right)=\frac{\left(\begin{array}{c}
K \\
m
\end{array}\right) \prod_{i=0}^{m-1}\left(\begin{array}{c}
N-i r \\
r
\end{array}\right) G(K-m, N-m r, r)}{K^{N}}
$$

where $m=0 \ldots K$ and

$$
\begin{aligned}
& G(M, l, v)= \\
& M^{l}+\sum_{i=1}^{\left\lfloor\frac{l}{v}\right\rfloor}\left\{(-1)^{i} \prod_{j=0}^{i-1}\left\{\left(\begin{array}{c}
l-j v \\
v
\end{array}\right)(M-j)\right\}(M-i)^{l-i v} \frac{1}{i !}\right\}
\end{aligned}
$$

Since all the acknowledged tags in a cycle withdraw from contention, the transition matrix $H$ and transition probabilities are given by

$$
h_{i j}=\left\{\begin{array}{l}
P_{K, N-i}\left(\mu_{1}=j-i\right), i<j \leq i+K \\
1-\sum_{k=i+1}^{i+K} h_{i, k}, i=j \\
0, \text { otherwise }
\end{array}\right.
$$

where $i=0 \ldots N$. Since this is an absorbing Markov chain, the average number of identification cycles equals the average number of steps to absorption, which is given by

$$
\mathbf{t}=F \mathbf{c}
$$

where $\mathbf{t}$ is a column vector and $t_{s}$ is the expected number of steps (cycles, in our case) before the chain is absorbed given that the chain starts in state $X_{s}, F$ is the fundamental matrix of $H$ and $\mathbf{c}$ is a column vector all of whose entries are 1 (see [11]). Thus, if the starting state is $X_{1}$, that is, all the $N$ tags to be identified, the average number of cycles to identify all the tags is $t_{1}$.

Table 1 shows the average number of cycles versus number of tags $(N)$ for different frame lengths (in number of slots). It shows that with a fixed 
framed length, the number of cycles increases exponentially with the number of tags. Therefore, a simple mechanism like framed slotted ALOHA does not scale well, requiring a frame adaptation mechanism as the number of tags increases.

\subsection{Carrier Sense Multiple Access}

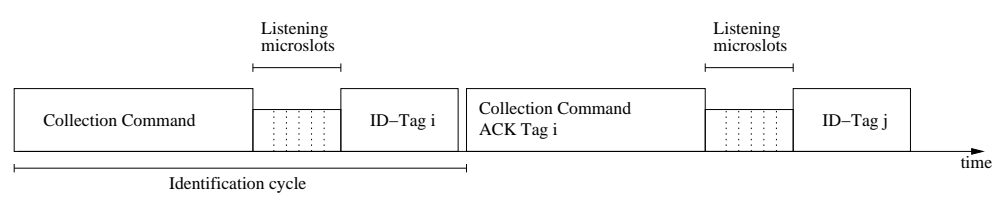

Fig. 2. Anti-collision procedure with CSMA

The operation of the identification protocol when using CSMA would be as follows: after receiving a collection command from the reader all $N$ tags listen to the channel for a number of micro-slots chosen randomly from a set of $K$. If the channel remains idle after the number of selected micro-slots, a node sends its ID. Otherwise, it withdraws until the next collection command. If there is no collision, the reader sends an ACK-Collection command, which indicates the node already identified and asks for more IDs. The remaining nodes start the process again. Fig. 2 illustrates this mechanism.

The probability of success $\pi_{p}(N)$ when $N$ nodes select a contention microslot using probability distribution $p$, where $p_{r}$ is the probability each contender independently picks slot $r$, is [9]:

$$
\pi_{p}(N)=N \sum_{s=1}^{K-1} p_{s}\left(1-\sum_{r=1}^{s} p_{r}\right)^{N-1}
$$

In this case, the transition matrix $H$ for the Markov process $\left\{X_{s}\right\}$ defined previously is

$$
h_{i j}=\left\{\begin{array}{l}
\pi_{p}(N-i), j=i+1 \\
1-\pi_{p}(N-i), i=j \\
0, \text { otherwise }
\end{array}\right.
$$

The average number of steps until absorption is computed as previously.

Let us assume first that the slots are chosen uniformly. In this case $p_{r}=\frac{1}{K}$. Fig. 3(a) and 3(b) show the results using a uniform distribution for different number of micro-slots. Again, the actual duration of an identification cycle depends on the number of micro-slots, the packet length and the transmission 


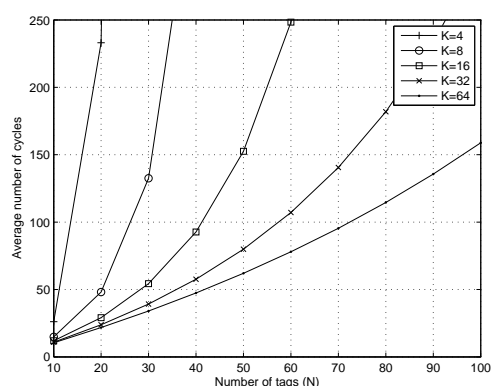

(a) Average number of cycles with uniform distribution

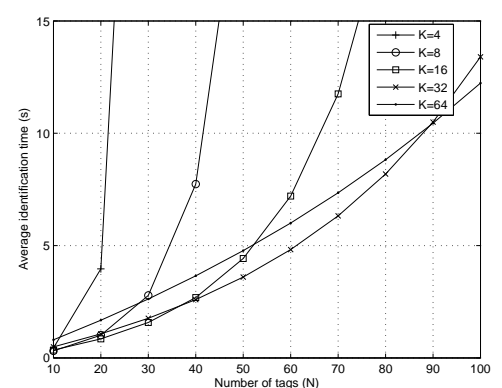

(b) Average time with uniform distribution

Fig. 3. Performance of identification process with uniform distribution

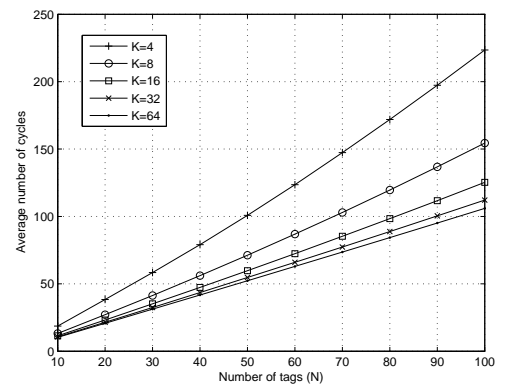

(a) Average number of cycles with Sift $(\mathrm{M}=64)$

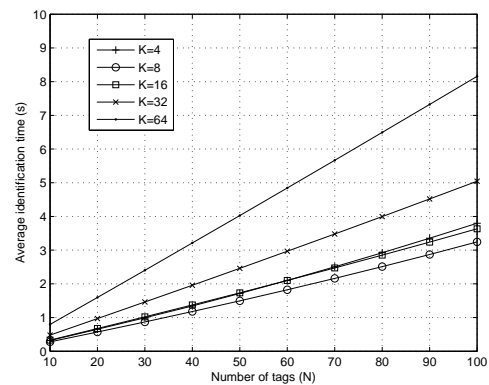

(b) Average time with Sift distribution

Fig. 4. Performance of identification process with Sift distribution

rate. The parameters for the computation of the average time are given in Section 4. Like framed slotted ALOHA, this procedure does not scale well either. In fact, its performance is worse and together with the additional device complexity it may be one of the reasons why it has never been proposed as an anti-collision procedure for RFID systems.

Let us assume now the Sift distribution is used, which is an approximation to the optimised distribution derived in reference [9]. In this case, $p_{r}=\frac{\alpha^{-r}(1-\alpha) \alpha^{K}}{1-\alpha^{K}}$ for $r=1 \ldots K$ and $\alpha=M^{\frac{-1}{K-1}} . M$ is a parameter of the Sift distribution, pre-configured before deployment and representing the maximum number of contenders (as expected by the designer). The results shown in Fig. 4(a) and 4(b) reveal that the number of cycles increases almost linearly with the number of tags, unlike the exponential increment of framed slotted ALOHA. Therefore, this procedure scales well. In addition, by increasing the number of micro-slots the number of cycles tends to the minimum necessary 
( $N$ cycles), but it implies increasing the duration of a cycle and may be even counterproductive: as seen in Fig. 4(b), 8 micro-slots are enough to handle the entire range of tags.

These results show that after choosing carefully the distribution for the contention window CSMA becomes an scalable technique for the identification of RFID tags. In Section 4, the different proposals for active tags are compared and discussed.

\section{Comparison of proposals}

In order to compare the different proposals the identification cycles are translated to absolute time, since the actual duration of an identification cycle depends on the number of slots. First, we compare non-persistent CSMA with Sift distribution versus ISO 18000-7. The parameters are chosen from the specification [5]. An identification cycle lasts a collection command (5 ms) plus each slot $(8 \mathrm{~ms})$. Finally, for each identified tag the interrogator sends an ACK packet (5 ms) before starting a new cycle. For the CSMA cycle, we assume the same duration for the interrogator commands $(5 \mathrm{~ms})$ and ID packets $(8 \mathrm{~ms})$ plus the time for all the micro-slots, though the expected successful slot comes earlier. In fact, the performance depends to a great extent on the minimum time needed to perform the carrier sense, that is, the duration of the contention micro-slot. The duration and accuracy of carrier sensing (Clear Channel Assessment, CCA) depends on the technology, device and implementation [12]. There are many possibilities, but we assume that devices use coherent CCA, that is, the channel is busy when the packet preamble is detected. Thus, we set the micro-slot time to the duration of preamble. For ISO 18000-7, preamble is around $1 \mathrm{~ms}$ and so it is the time per microslot. Indeed it can be considered a conservative value, since current devices can perform this task in less time [12]. Even though, as can be seen in Fig. 5, non-persistent Sift-based CSMA with 8 micro-slots (and Sift parameter $\mathrm{M}=64$ ) outperforms the procedure proposed by ISO 18000-7 for every frame length. When the number of tags is low, the improvement is not significative, but, as the number increases, framed slotted ALOHA becomes unstable and the frame length must be adapted. On the contrary, non-persistent Sift-based CSMA can handle all the range of tags seamlessly.

Finally, we compare with EPC "Gen 2". In this case, according to the specification [7], empty slots and slots with collision are shorter than slots with correct tag ID packets. However, we provide an approximation of the average identification time, assuming that the duration of all slots is the same and equals $2.505 \mathrm{~ms}$, which is the time needed for the correct identification of a single tag at $40 \mathrm{Kbps}$. Thus, this is a conservative estimate, since empty and collision slots are actually shorter $(0.575 \mathrm{~ms})$. For CSMA, we again assume that in a cycle we have a tag ID packet $(1.4 \mathrm{~ms})$ plus an interrogator ACKCollection $(0.55 \mathrm{~ms})$, plus the duration of the entire contention window, as 


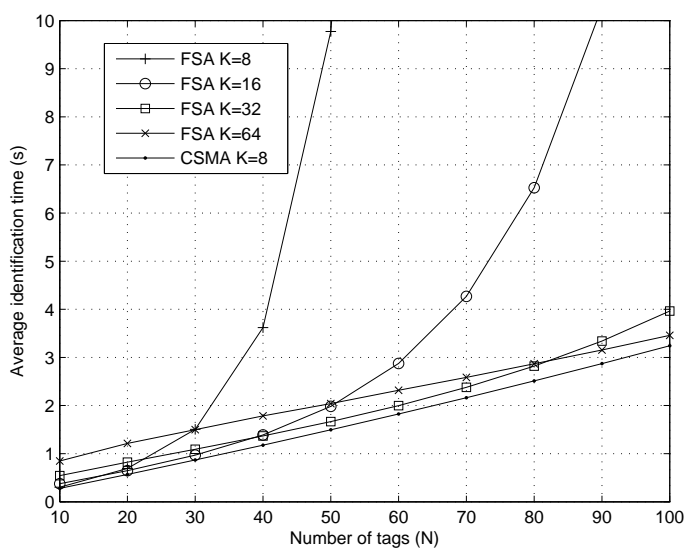

Fig. 5. Comparison of ISO 18000-7 versus CSMA with Sift distribution

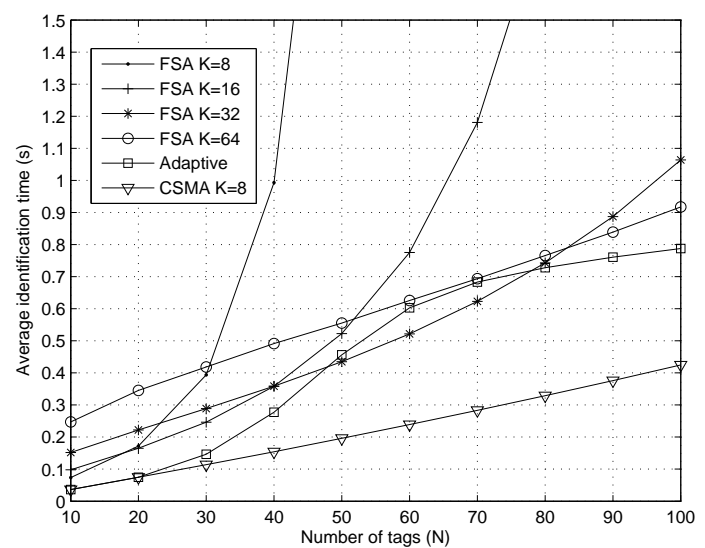

Fig. 6. Comparison of EPC "Gen 2" versus CSMA with Sift distribution

before. In this case, we set the micro-slot time to $100 \mu \mathrm{s}$, which is again the duration of the preamble. In Figure 6 we depict the results for EPC and nonpersistent Sift-based CSMA $(\mathrm{M}=64)$. In addition to the average number of cycles previously computed, we have simulated the EPC frame adaptation mechanism recommended in the specification [7]. It is also included in Figure 6 labelled as "Adaptive". Obviously, for fixed frame length the results are the same as in Fig. 5 but with another time scale. However, this figure shows in addition that non-persistent Sift-based CSMA outperforms EPC with frame adaptation as well. Moreover, in this case, the improvement is even better (around $50 \%$ for almost every number of tags) due to the shorter micro-slot, as discussed before. 
In summary, CSMA allows for a quicker identification of tags in all the cases. The actual improvement depends on the duration of the contention micro-slot. However, it is more important to remark that CSMA also scales much better than framed slotted ALOHA, even with frame adaptation, which simplifies the implementation of reader and tags.

\section{Conclusions}

In this paper we propose the use of non-persistent Sift-based CSMA as an anti-collision procedure for RFID active tags. Current proposals directly use the approach for passive tags, that is, framed slotted ALOHA, which does not scale well and needs an additional procedure for frame adaptation. However, active devices already include carrier sense capabilities with no extra cost and, thus, CSMA may be used seamlessly. In fact, compared to some commercial products that use more sophisticated protocols, like IEEE 802.11 or Bluetooth, CSMA may even reduce the cost of active devices while achieving the goal of the anti-collision procedure.

To support our proposal, we have evaluated the average time nedeed for identification with this mechanism and compared it with the current ISO 18000-7 and EPC "Gen 2" standards. The results show that CSMA outperforms both of them. For instance, the average identification time can be decreased by $50 \%$ compared to EPC with frame adaption. In fact, the performance improvement depends on the duration of the contention microslots. More important is the fact that, in both cases, non-persistent Sift-based CSMA also scales much better: configured with 8 micro-slots CSMA can effectively handle a range that spans from a few tags to hundreds of them, without the need for additional adaptation mechanisms, which simplifies the implementation of reader and tags. Even though, the number of contention microslots may also be adapted if necessary. As future work we are currently investigating adaptation mechanisms for CSMA as well as different techniques to optimise the frame length of slotted ALOHA.

\section{Acknowledgements}

This work has been funded by the Spanish Ministerio de Educación y Ciencia with the projects DEP2006-56158-C03-03/EQUI and m:ciudad (FIT-3305032006-2, partially funded by ERDF) and by the Spanish Research Council with the ARPaq project (TEC2004-05622-C04-02/TCM). 


\section{References}

1. Stanford, V., "Pervasive Computing Goes the Last Hundred Feet with RFID Systems", IEEE Pervasive Computing, vol. 2, no. 2, pp. 9-14, AprilJune 2004.

2. Shih, D., Sun, P., Yen, D., Huang, S., "Taxonomy and survey of RFID anti-collision protocols", Elsevier Computer Communications, vol. 29, pp. 2150-2166, 2006.

3. Vogt, H., "Efficient Object Identification with Passive RFID Tags", Lecture Notes in Computer Science, vol. 2414, pp. 98-113, 2002.

4. Zhou, F., Chen, C., Jin, D., Huang, C., Min, H., "Evaluating and Optimizing Power Consumption for Anti-Collision Protocols for Applications in RFID Systems", in Proc. Int. Symp. on Low Power Electronics and Design 2004, pp. 357-362, 2004.

5. ISO/IEC 18000-7:2004 Information technology-Radio frequency identification for item management-Part 7: Parameters for active air interface at 433 $\mathrm{MHz}, 2004$.

6. Wieselthier, J. E., Ephremides, A., Michaels, L. A., "An exact analysis and performance evaluation of framed ALOHA with capture", IEEE Transactions on Communications, vol. 37(2), pp. 125-137, 1988.

7. Class 1 Generation 2 UHF Air Interface Protocol Standard Version 1.0.9: "Gen 2". Available online at: http://www.epcglobalinc.org/standards

8. Zhao, F. y Guibas, L., Wireless Sensor Networks. An information processing approach. Morgan Kaufmann, 2004.

9. Tay, Y., C., Jamieson, K., Balakrishnan, H., "Collision-Minimizing CSMA and its Applications to Wireless Sensor Networks", IEEE Journal on Selected Areas in Communications, vol. 22(6), pp. 1048-1057, 2004.

10. Kleinrock, L., Tobagi, F., "Packet Switching in Radio Channels: Part ICarrier Sense Multiple Access and their throughput-delay characteristics", IEEE Transactions on Communications, vol. 23, pp. 1400-1416, 1975.

11. Grinstead, C. M., Snell, J. L., Introduction to Probability, 2nd Edition, American Mathematical Society, 2003.

12. Ramachandran, I., Das, A., Roy, S., "Clear Channel Assessment in Energyconstrained Wideband Wireless Networks", IEEE Wireless Communications Magazine, forthcoming. 